BNL -67849

\title{
AN ENERGY RECOVERY ELECTRON LINAC-ON-RING COLLIDER
}

L. Merminga, G. A. Krafft and V.A. Lebedev Jefferson Laboratory

12000 Jefferson Avenue

Newport News, VA 23606, USA

Ilan Ben-Zvi

National Synchrotron Light Source

Brookhaven National Laboratory

P.O. Box 5000

Upton, New York 11973-5000, USA

September 2000

\author{
National Synchrotron Light Source \\ Brookhaven National Laboratory \\ Operated by \\ Brookhaven Science Associates \\ Upton, NY 11973
}

Under Contract with the United States Department of Energy

Contract Number DE-AC02-98CH10886 


\section{DISCLAIMER}

This report was prepared as an account of work sponsored by an agency of the United States Government. Neither the United States Government nor any agency thereof, nor any of their employees, nor any of their contractors, subcontractors or their employees, makes any warranty, express or implied, or assumes any legal liability or responsibility for the accuracy, completeness, or any third party's use or the results of such use of any information, apparatus, product, or process disclosed, or represents that its use would not infringe privately owned rights. Reference herein to any specific commercial product, process, or service by trade name, trademark, manufacturer, or otherwise, does not necessary constitute or imply its endorsement, recommendation, or favoring by the United States Government or any agency thereof or its contractors or subcontractors. The views and opinions of authors expresses herein do not necessarily state to reflect those of the United States Government or any agency thereof. 


\title{
An Energy Recovery Electron Linac-On-Ring Collider
}

\author{
L. Merminga*, G. A. Krafft*, V. A. Lebedev*, and Ilan Ben-Zvi ${ }^{\dagger}$ \\ *Jefferson Laboratory, 12000 Jefferson Ave., Newport News, VA 23606, USA \\ ${ }^{\dagger}$ Brookhaven National Laboratory, P. O. Box 5000, Upton, NY, 11973-5000, USA ${ }^{l}$
}

\begin{abstract}
We present the design of high-luminosity electron-proton/ion colliders in which the electrons are produced by an Energy Recovering Linac (ERL). Electronproton/ion colliders with center of mass energies between $14 \mathrm{GeV}$ and $100 \mathrm{GeV}$ (protons) or $63 \mathrm{GeV} / \mathrm{A}$ (ions) and luminosities at the $10^{33}$ (per nucleon) level have been proposed recently as a means for studying hadronic structure. The linac-on-ring option presents significant advantages with respect to: 1) spin manipulations 2) reduction of the synchrotron radiation load in the detectors 3) a wide range of continuous energy variability. Rf power and beam dump considerations require that the electron linac recover the beam energy. Based on extrapolations from actual measurements and calculations, energy recovery is expected to be feasible at currents of a few hundred $\mathrm{mA}$ and multi-GeV energies. Luminosity projections for the linacring scenario based on fundamental limitations are presented. The feasibility of an energy recovery electron linac-on-proton ring collider is investigated and four conceptual point designs are shown corresponding to electron to proton energies of: $3 \mathrm{GeV}$ on $15 \mathrm{GeV}, 5 \mathrm{GeV}$ on $50 \mathrm{GeV}$ and $10 \mathrm{GeV}$ on $250 \mathrm{GeV}$, and for gold ions with $100 \mathrm{GeV} / \mathrm{A}$. The last two designs assume that the protons or ions are stored in the existing RHIC accelerator. Accelerator physics issues relevant to proton rings and energy recovery linacs are discussed and a list of required R\&D for the realization of such a design is presented.
\end{abstract}

\section{INTRODUCTION}

Electron-proton/ion colliders with center of mass energies between $14 \mathrm{GeV}$ and 100 $\mathrm{GeV}$ (protons) or $63 \mathrm{GeV} / \mathrm{A}$ (ions) and luminosities at the $10^{33}$ (per nucleon) level have been proposed recently as a means for studying hadronic structure. Electron beam polarization appears to be crucial for many of the experiments. Two

\footnotetext{
${ }^{1}$ Done under the auspices of the U.S. Department of Energy under contract number DE-AC0298CH10886
} 
accelerator design scenarios have been examined in detail: colliding rings and recirculating linac-on-ring. Although the linac-on-ring scenario is not as well developed as the ring-ring scenario, comparable luminosities appear feasible. The linac-on-ring option presents significant advantages with respect to: 1) spin manipulations 2) reduction of the synchrotron radiation load in the detectors 3) a wide range of continuous energy variability. $\mathrm{Rf}$ power and beam dump considerations require that the electron linac recover the beam energy. This technology has been demonstrated at Jefferson Lab's IR FEL with cw current up to 5 $\mathrm{mA}$ and beam energy up to $50 \mathrm{MeV}$. Based on extrapolations from actual measurements and calculations, energy recovery is expected to be feasible at higher currents (a few hundred $\mathrm{mA}$ ) and higher energies (a few $\mathrm{GeV}$ ) as well.

We begin with a brief overview of Jefferson Lab's experience with energy recovery and summarize its benefits. Luminosity projections for the linac-ring scenario based on fundamental limitations are presented next. The feasibility of an energy recovery electron linac-on-proton ring collider is investigated and four conceptual point designs are shown corresponding to electron to proton energies of: $3 \mathrm{GeV}$ on $15 \mathrm{GeV}, 5 \mathrm{GeV}$ on $50 \mathrm{GeV}$ and $10 \mathrm{GeV}$ on $250 \mathrm{GeV}$, and for gold ions with $100 \mathrm{GeV} / \mathrm{A}$. The last two designs assume that the protons or ions are stored in the existing RHIC accelerator. Accelerator physics issues relevant to proton rings and energy recovery linacs are discussed next and a list of required R\&D for the realization of such a design is presented. 


\section{Energy Recovery Linacs}

Energy recovery is the process by which the energy invested in accelerating a beam is returned to the rf cavities by decelerating the beam. To date, energy recovery has been realized in a number of different ways. The first energy recovery experiment was done at Stanford's superconducting rf (srf) linac where the recirculated electron beam was injected into the linac in such a phase that it lost energy to the cavity fields [1]. This is an example of the so-called same-cell energy recovery. All of the linac beam energy was recovered. Another experimental demonstration of energy recovery took place at Los Alamos where $>70 \%$ of the beam energy was recovered [2]. Following acceleration, the beam was transported around a $180^{\circ}$ bend and passed through a decelerating structure. The decelerators were coupled to the accelerators through the resonant bridge couplers. The rf power generated by the beam was shared with the accelerators through the couplers. An energy recovery experiment was also performed in the CEBAF Injector. Energy recovery was demonstrated in $\mathrm{cw}$ mode and at the same time multipass Beam Breakup (BBU) experiments were carried out [3].

Same-cell energy recovery with cw current up to $5 \mathrm{~mA}$ and energy up to $50 \mathrm{MeV}$ has been demonstrated at Jefferson Lab's (JLab) IR FEL and it is used routinely for the operation of the FEL as a User Facility [4]. The machine layout is shown in Figure 1. Microbunches with an rms bunch length of $\sim 20 \mathrm{psec}$ are produced in a DC photocathode gun and accelerated to $320 \mathrm{kV}$. The bunches are compressed by a copper buncher cavity operating at $1497 \mathrm{MHz}$. They pass through a pair of srf cavities operating at an average gradient of $10 \mathrm{MV} / \mathrm{m}$. The output beam at $\sim 10 \mathrm{MeV}$ is injected into an 8-cavity srf cryomodule where it is accelerated up to $\sim 48 \mathrm{MeV}$. The beam then passes through the wiggler. Afterward it is recirculated through two isochronous, achromatic bends separated by a quadrupole transport line, back through the cryomodule in the decelerating rf phase and dumped at the injection energy of $\sim 10 \mathrm{MeV}$.

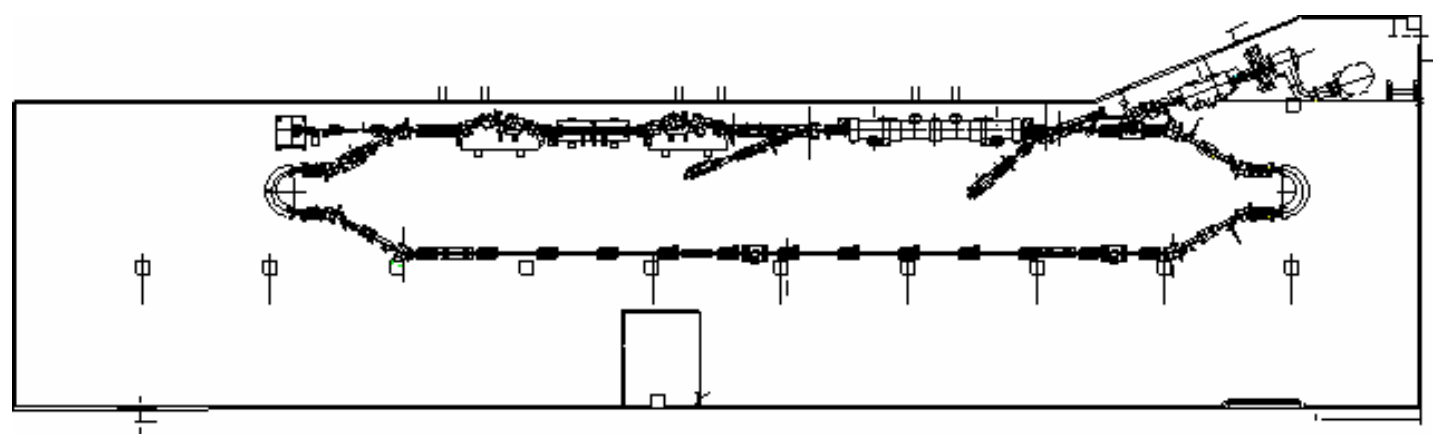


Figure 1. Machine layout of Jefferson Lab's IR FEL.

The first indicators of energy recovery, which subsequently were used as diagnostic tools, are the klystron drive signals for the gradient feedback loop, shown in Figure 2, for four of the linac rf cavities. When a $200 \mu \mathrm{sec}$ beam pulse is injected in these cavities, in the absence of energy recovery, the gradient drive signals reach $\sim 2 \mathrm{~V}$ to compensate for beam loading. With energy recovery, these signals are close to $0 \mathrm{~V}$ (where $0 \mathrm{~V}$ corresponds to the $\mathrm{DC}$ voltage required to drive the accelerating field in the cavity), as the decelerating and accelerating beam vectors cancel each other resulting in nearly zero net beam loading.

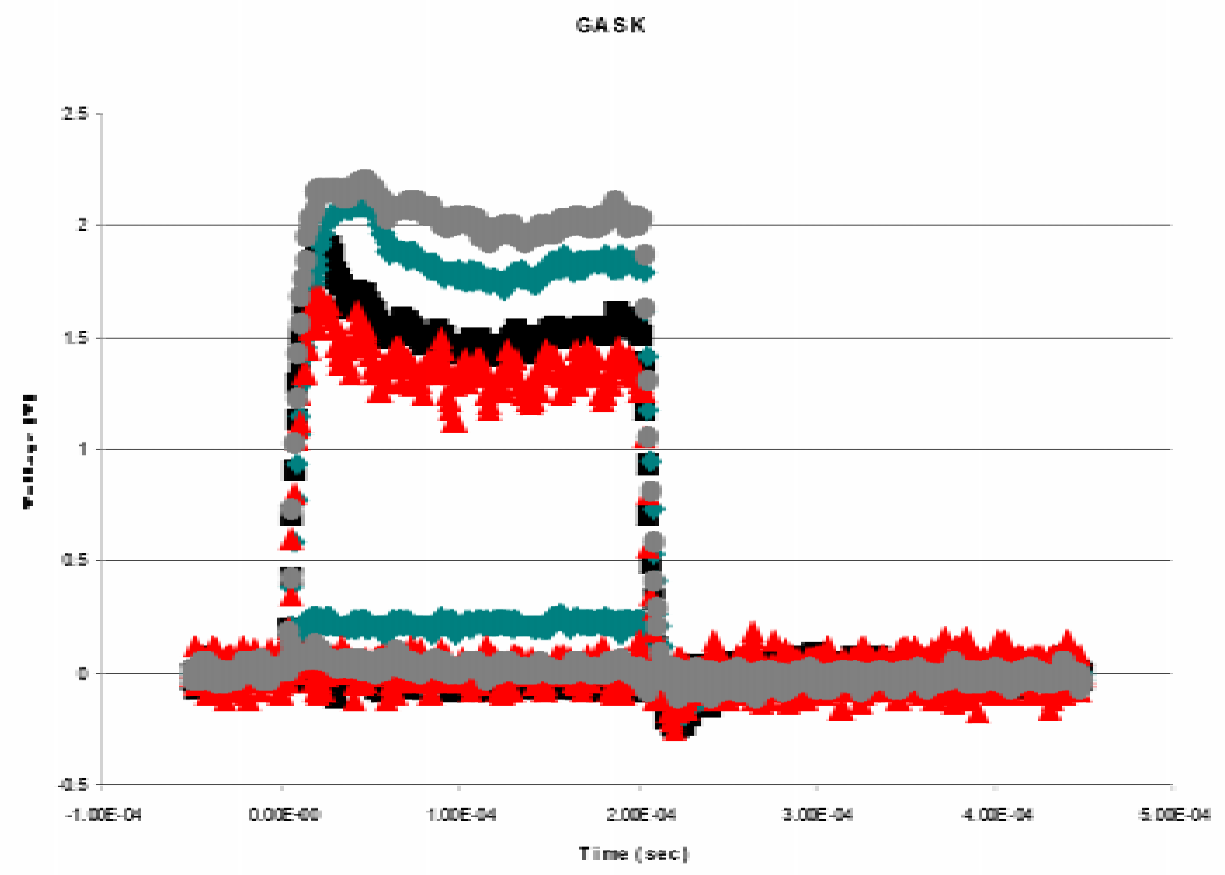

Figure 2. Response of the gradient loop drive signals, in four rf cavities, to a $200 \mu$ sec beam pulse, with and without energy recovery.

Another indication that energy recovery works is demonstrated in Figure 3 where the rf power required to accelerate up to $3.5 \mathrm{~mA}$ of $\mathrm{cw}$ beam current is compared to the power required for no beam, in each of the 8 cavities. Notice that the required $\mathrm{rf}$ power is nearly independent of beam current. An additional benefit is that the overall system efficiency is increased. 


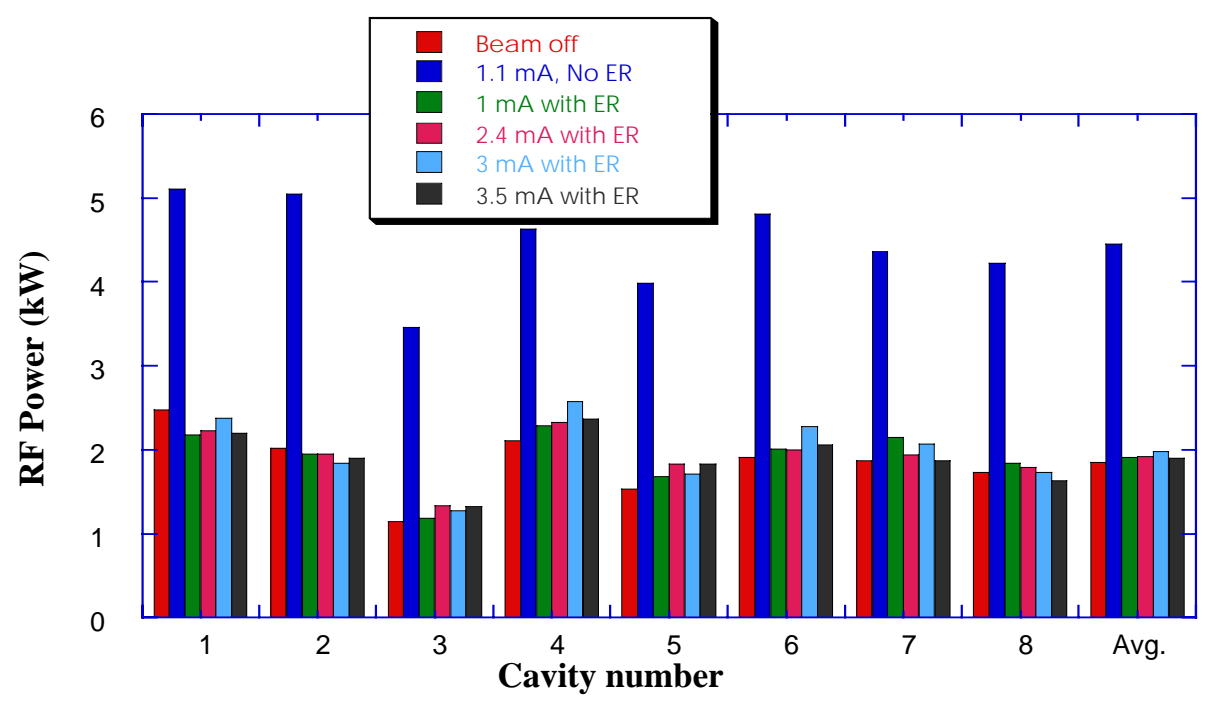

Figure 3. Rf power requirements in the linac cavities for a range of beam currents.

The HERA electron storage ring in DESY circulates a $27 \mathrm{GeV}$ electron beam through a large number of superconducting cavities at an average current of $40 \mathrm{~mA}$. While this is not strictly an energy recovery system (and not a linac), it provides a demonstration that a high energy, high current beam can be circulated through a very large number of passes in a long superconducting structure.

In summary, the benefits of energy recovery are:

1) The required rf power becomes nearly independent of beam current.

2) The overall system efficiency is increased.

3) The electron beam power to be disposed of at the beam dumps is reduced by the ratio of the final to injected energy.

4) The induced radioactivity (and therefore the shielding problem) is reduced, if the beam is dumped below the neutron production threshold.

\section{Fundamental Limitations Of Colliders}

The luminosity of an electron linac-on-proton ring collider, assuming both beams are round gaussians at the interaction point (IP), is given by

$$
L=\frac{N^{N} p}{4 \pi \sigma^{* 2}} f_{c}
$$


where $N_{e}$ is the number of electrons per bunch, $N_{p}$ is the number of protons per bunch, $f_{c}$ is the bunch collision frequency, and $\sigma^{*}$ is the rms beam size at the IP. The luminosity is proportional to the product of the intensity of one beam $N f_{c} / \varepsilon$, and the number of particles per bunch in the other beam, where $\varepsilon$ is the beam emittance. A number of effects impose fundamental limitations on the intensity, which together with limitations on the number of particles per bunch can ultimately limit the luminosity. We discuss intensity limits next.

\section{a) Luminosity at the Laslett and Beam-Beam Tuneshifts Limit}

The Laslett tuneshift $\Delta v_{L}$ is given by:

$$
\Delta v_{L}=\frac{N_{p}}{\varepsilon_{p} \sigma_{z}^{p}} \frac{r_{p}}{4 \pi \gamma_{p}^{3}} \frac{C}{\sqrt{2 \pi}}
$$

where $C$ is the ring circumference and $r_{p}$ the classical radius of proton $1.534 \times 10^{-18}$ m. Typically, in proton ring designs, the bunch length is chosen to be approximately equal to the beta function at the IP in order for the luminosity not to deteriorate too much within one collision. In this approximation, one concludes that the Laslett tuneshift imposes a fundamental limit on the ratio of $N_{p} / \sigma_{p}^{* 2}$.

The beam-beam tuneshift of the proton beam given by:

$$
\xi_{p}=\frac{N_{e} r_{p} \beta_{p}^{*}}{4 \pi \gamma_{p} \sigma_{e}^{* 2}}
$$

where $\beta_{p}^{*}$ is the beta function at the IP, also imposes a fundamental limit on $N_{e} / \sigma_{e}^{* 2}$. One can write an expression for the luminosity in the limit of Laslett and beam-beam tuneshifts:

$$
L=\left(\frac{4 \pi \sqrt{2 \pi}}{r_{p}^{2}}\right) \xi_{p} \Delta v_{L} \frac{\gamma_{p}^{4}}{C} \frac{\sigma^{* 2}}{\beta^{*}} f_{c}
$$

As an example, we can assume a beta function at the IP $\beta^{*}=10 \mathrm{~cm}$, an rms beam size at the IP $\sigma^{*}=40 \mu \mathrm{m}$ and collision frequency $f_{c}=150 \mathrm{MHz}$. Figure 4 is the plot of luminosity vs. proton beam energy $\mathrm{E}_{\mathrm{p}}$, given by Eq. (4). The ring circumference $C$ has been minimized subject to the engineering constraint of maximum magnetic field 
(in this case $\mathrm{B}=4$ Tesla). The two curves correspond to: a) $\Delta v_{L}=0.004$, which is a safe and generally accepted value for the Laslett tuneshift and b) $\Delta v_{L}=0.04$, which is a more aggressive value, yet consistent with the value assumed in the ring-ring scenario presented in this document [5].

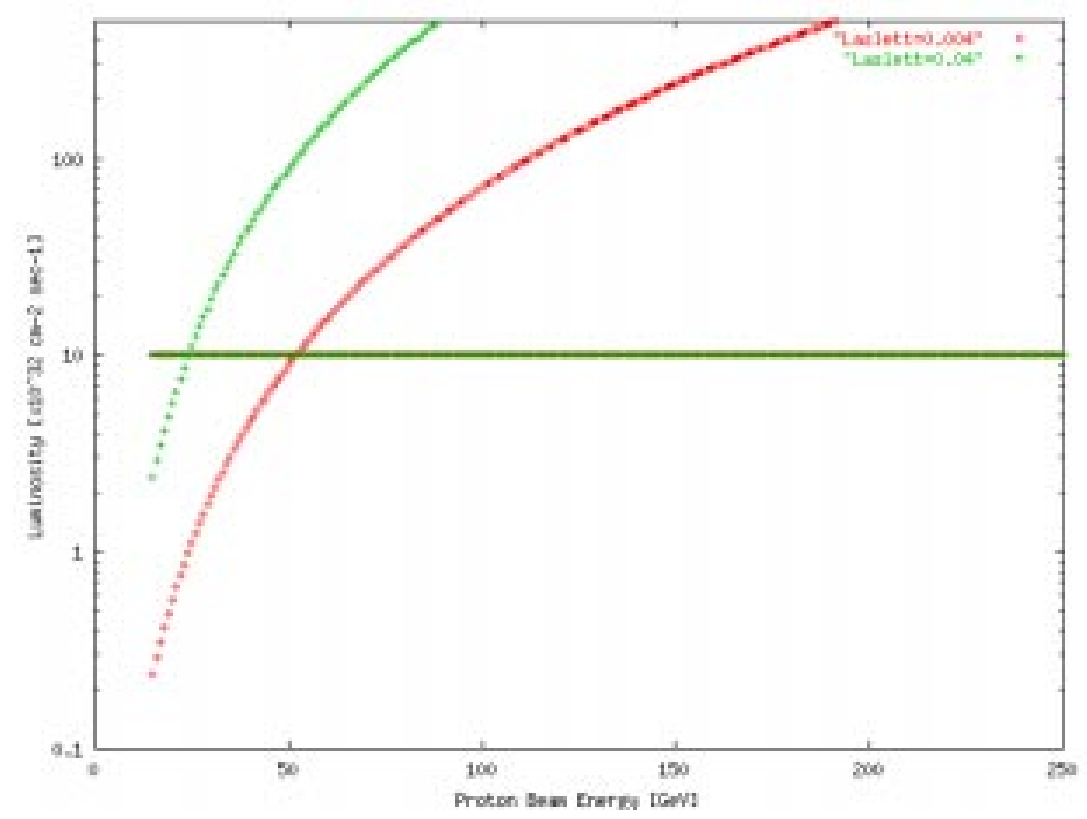

Figure 4. Luminosity vs. proton beam energy at the Laslett and beam-beam tuneshift limits, for two values of the Laslett tuneshift: 0.004 and 0.04 . In both cases the beam-beam tuneshift is 0.004 .

In both cases $\xi_{p}=0.004$, consistent both with [5] and with the value assumed in the eRHIC ring-ring scenario [6]. Of course, in practice $\mathrm{N}_{\mathrm{p}}$ and $\mathrm{N}_{\mathrm{e}}$ are further limited by a number of other effects such as collective instabilities, and we will examine these later. The horizontal line in Fig. 4 corresponds to luminosity equal to $1.0 \times 10^{33} \mathrm{~cm}^{-2} \mathrm{sec}^{-1}$.

\section{b) Luminosity at the Beam-Beam Induced Head-Tail Instability Limit}

The beam-beam induced head-tail instability is an additional effect, which could potentially impose a limit on the luminosity of linac-ring colliders. Presently this instability is the subject of focused investigation at Jefferson Lab. The effect is analyzed later on in this report, using a linearized model, according to which the stability condition can be expressed as

$$
D_{e} \xi_{p} \leq 4 v_{s}
$$


where $D_{e}$ is the electron beam disruption parameter given by

$$
D_{e}=\frac{N_{p} r_{e} \sigma_{z}^{p}}{\gamma_{e} \sigma_{p}^{* 2}}
$$

and $v_{s}$ is the synchrotron tune of the proton beam. One can re-write the luminosity in the limit of the head-tail instability, as

$$
L=\left(\frac{4}{r_{e} r_{p}}\right) \gamma_{e} \gamma_{p} \nu_{s} \sigma^{* 2} f_{c} .
$$

Figure 5 displays the luminosity given by Eq. (7) as function of proton energy for four values of the electron beam energy: $3,5,7,10 \mathrm{GeV}$. The synchrotron tune has been set equal to $1 \times 10^{-3}$, the rms angular divergence of the beam at the IP, $\sigma^{* *}=\sigma^{*} / \beta^{*}=40 \mu \mathrm{m} / 10 \mathrm{~cm}=0.4 \mathrm{mrad}$ and the collision frequency $f_{c}=150 \mathrm{MHz}$. Superimposed are the luminosity curves from Figure 4 for comparison. Figure 5 demonstrates that above a certain proton beam energy, increasing the Laslett tuneshift beyond the generally accepted values does not benefit the luminosity. A second conclusion is that, assuming that the larger value of the Laslett tuneshift is attainable, then the luminosity is limited by the head-tail instability, over most of the energy range of protons and electrons.

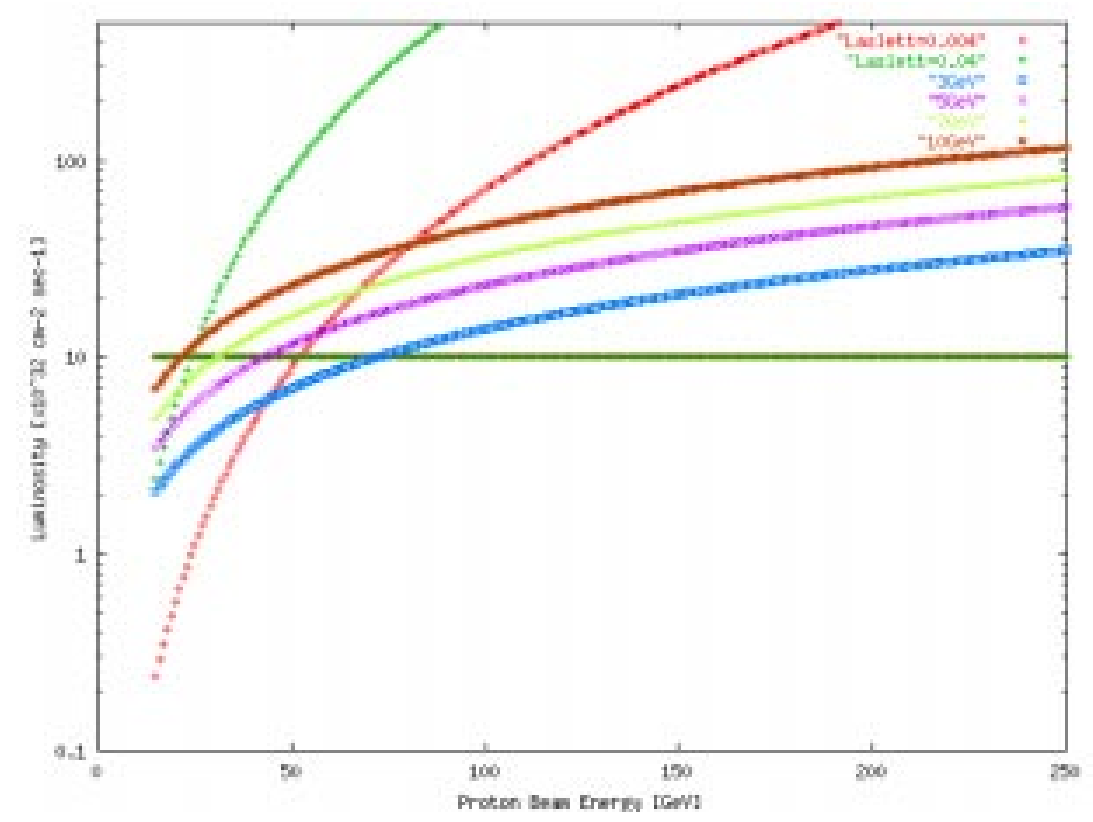


Figure 5. Luminosity vs. proton beam energy, at the stability limit of the beam-beam induced headtail effect (linear approximation).

The above expressions ought to be compared to the luminosity expressions for a ring-ring collider based on fundamental limitations. In a ring-ring design these are the beam-beam tuneshifts of both electrons and protons. The luminosity in the beambeam tuneshift limit is given by:

$$
L=\left(\frac{4 \pi}{r_{e} r_{p}}\right) \gamma_{e} \gamma_{p} \xi_{e} \xi_{p} \sigma^{* 2} f_{c}
$$

Ring-ring limitations and comparisons with linac-ring will be the subject of a follow-up article.

\section{Conceptual Point Designs}

We now turn our attention to specific point designs that span a proton energy range from 15 to $250 \mathrm{GeV}$ and an electron energy range from 3 to $10 \mathrm{GeV}$. As was discussed above, the head-tail instability is presently under investigation. The condition presented earlier was derived using a linear approximation, which clearly does not reflect the true complexity of the problem. The next steps include a more sophisticated analysis and simulation studies. In the absence of more rigorous results, we will develop a reasoning for the point designs without taking the head-tail instability into account and will defer the more complete study to a later document.

We present reasoning that allows us to develop a self-consistent sets of parameters. Four sets of input parameters will be considered: a) Proton beam energy of $15 \mathrm{GeV}$ colliding with electron beam energy of $3 \mathrm{GeV}$ b) Proton beam energy of $50 \mathrm{GeV}$ and electron beam energy of $5 \mathrm{GeV}$ c) proton beam energy of $250 \mathrm{GeV}$ and electron beam energy of $10 \mathrm{GeV}$ d) gold ion beam of $100 \mathrm{GeV} / \mathrm{A}$ and electron beam energy of $10 \mathrm{GeV}$. The high-energy designs are based on the existing RHIC storage ring.

Since the linac technology assumed is the same for all designs, we will briefly discuss it here. The technology of the electron linac is well established. Jefferson Laboratory has significant expertise in srf linacs and the srf accelerating structures are commercially available from a number of manufacturers.

For the sake of this report we assume that the linac structures will be identical to the well-known TESLA style cavities. These cavities and the ancillary equipment (cryostats, couplers, tuners, HOM loads etc.) have been optimized cost-wise and performance-wise. The TESLA cavities have a shunt impedance $\mathrm{R} / \mathrm{Q}=1036 \mathrm{Ohms}$ and structure length $1.038 \mathrm{~m}$. The residual resistance is $3 \mathrm{n} \Omega$, equivalent to a $\mathrm{Q}$ of $10^{11}$. Considering demonstrated performance from a number of manufacturers, we 
will assume, conservatively, a $\mathrm{Q}_{0}$ of $1.5 \times 10^{10}$ at $2 \mathrm{~K}$ and accelerating gradient of $20 \mathrm{MV} / \mathrm{m}$.

At these values the refrigeration power is $26 \mathrm{~W} /$ structure. Thus a $10 \mathrm{GeV}$ linac, for example, will require 500 cavities with a dissipation (excluding standing losses) of $13 \mathrm{~kW}$. TESLA optimization was driven towards high gradient, not low Q. We

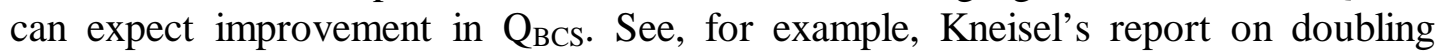
$\mathrm{Q}_{\mathrm{BCS}}$ by furnace baking in Ref. [7]. Figure 6 demonstrates the performance of TESLA style cavities.
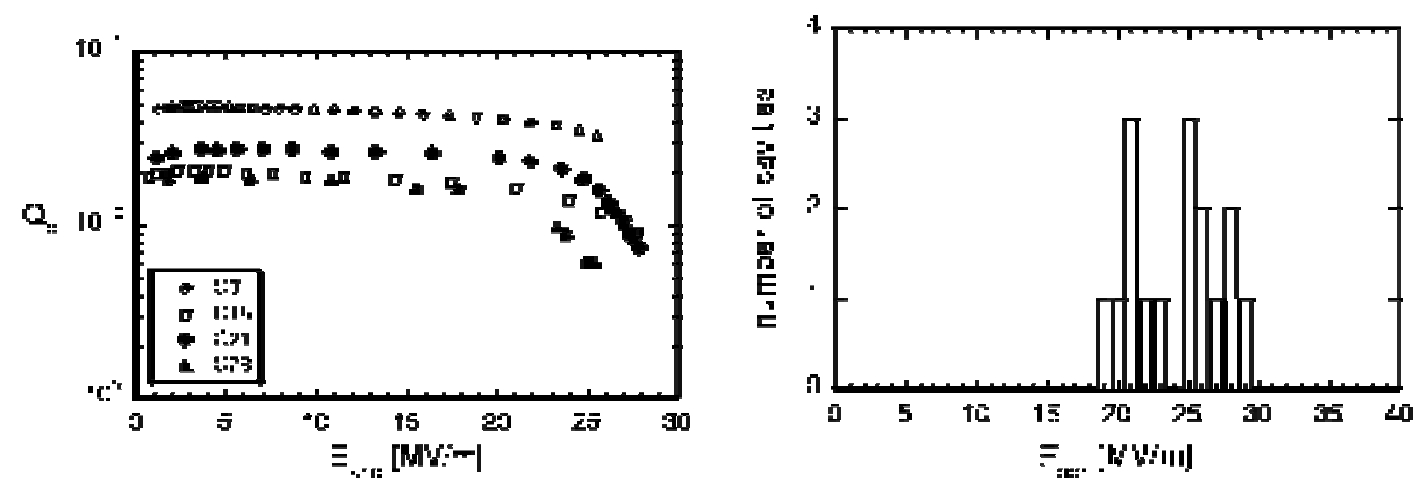

Figure 6. Plots of $\mathrm{Q}_{0}$ vs. accelerating gradient and distribution of gradients in the Tesla 9-cell cavities.

\section{1. $3 \mathrm{GeV}$ electrons on $15 \mathrm{GeV}$ protons}

\section{A: $\Delta v_{L} \leq 0.004$}

In this case we assume that both the Laslett and beam-beam tuneshifts can not exceed 0.004. To arrive at a self-consistent set of parameters and a luminosity estimate, we first set the electron beam size at the IP based on projected electron source performance. Then the proton beam parameters are set at the Laslett tuneshift limit. The maximum number of electrons per bunch is determined at the beam-beam tuneshift limit of the protons. Finally effects that influence the choice of the bunch collision frequency are discussed.

An rms normalized emittance of $60 \mu \mathrm{m}$ for electrons at a bunch charge of approximately $1 \mathrm{nC}$ is assumed, yielding a geometric emittance of $10 \mathrm{~nm}$ at the IP (3 $\mathrm{GeV}$ ). For a beta function of $12 \mathrm{~cm}$, which is discussed later, the rms electron beam size at the IP is $35 \mu \mathrm{m}$. (Round beams are assumed for electrons and protons.)

In order for the luminosity not to degrade, typically the beta function for the proton beam at the IP is set approximately equal to the rms proton bunch length. In this approximation, the Laslett tuneshift given by Eq. (2) can be written as 


$$
\Delta v_{L}=\frac{N_{p}}{\sigma_{p}^{* 2}} \frac{r_{p}}{4 \pi \gamma_{p}^{3}} \frac{C}{\sqrt{2 \pi}} .
$$

Clearly the tuneshift sets a limit on the ratio of $N_{p} / \sigma_{p}^{* 2}$. Assuming a proton beam rms normalized emittance of $3 \mu \mathrm{m}$ (consistent with LHC and RHIC experience) and $\Delta v_{L}=0.004$, the rms beam size for protons at the IP is $107 \mu \mathrm{m}$, for a beta function of $6 \mathrm{~cm}$. Then the number of protons per bunch at the Laslett tuneshift limit is $3 \times 10^{10}$.

The number of electrons per bunch can be limited either by the beam-beam tuneshift of the proton beam or by single-bunch transverse Beam Breakup in the linac [8]. Beam-beam tuneshift of the protons is given by Eq. (3), and we assume that it can not exceed 0.004. This value of the beam-beam tuneshift sets the number of electrons per bunch equal to $1.1 \times 10^{10}$. One can obtain a simple estimate for the emittance growth due to single bunch $\mathrm{BBU}$ in the linac by using the following expression [8],

$$
\eta=\frac{\operatorname{Lr}_{e} N_{e} W_{0}}{k_{0}\left(\gamma_{f}-\gamma_{0}\right)} \ln \frac{\gamma_{f}}{\gamma_{0}}
$$

where $k_{0}$ is the betatron wavenumber, $W_{0}$ is the transverse wake function, and $r_{e}$ the classical radius of the electron. For an rms bunch length of $1 \mathrm{~mm}$ and betatron wavelength in the linac of $50 \mathrm{~m}$, the amplification parameter $\eta$ remains less than $\sim 1$ if the number of electrons per bunch does not exceed $1.5 \times 10^{11}$. Should this effect become a serious limit, BNS damping can be used. Therefore, in this case the limit on $\mathrm{N}_{\mathrm{e}}$ is set by the beam-beam tuneshift, and not the single-bunch BBU.

The bunch collision frequency should be maximized subject to the constraints of parasitic collisions, user requirements and possibly the electron cloud effect in the proton ring. We have assumed a bunch separation of $6.66 \mathrm{nsec}$ or $150 \mathrm{MHz}$ repetition rate. Note that the luminosity scales linearly with the frequency.

For the case of unequal electron-proton bunch sizes, the luminosity is given by

$$
L=\frac{N_{e} N_{p} f_{c}}{2 \pi\left[\sigma_{e}^{* 2}+\sigma_{p}^{* 2}\right]} \text {. }
$$

For $\mathrm{N}_{\mathrm{e}}=1.1 \times 10^{10}, \mathrm{~N}_{\mathrm{p}}=3.0 \times 10^{10}, \mathrm{f}_{\mathrm{c}}=150 \mathrm{MHz}, \sigma_{\mathrm{e}}^{*}=35 \mu \mathrm{m}$ and $\sigma_{\mathrm{p}}^{*}=107 \mu \mathrm{m}$, the luminosity is equal to $6.2 \times 10^{31} \mathrm{~cm}^{-2} \mathrm{sec}^{-1}$.

IB: $\Delta v_{L} \leq 0.05$ 
We now consider a point design assuming that the Laslett tuneshift can be as high as 0.05 . In this case, the electron beam parameters remain the same and again the Laslett tuneshift sets the ratio of $N_{p} / \sigma_{p}^{* 2}$. However the optimization now proceeds as follows: We first determine the limit on $\mathrm{N}_{\mathrm{p}}$ and then set the minimum spot size at the IP, at the Laslett tuneshift limit.

The number of protons per bunch can be limited by collective instabilities or by the emittance growth of the electron beam due to a single round-beam collision with the protons. We set $\mathrm{N}_{\mathrm{p}}=1 \times 10^{11}$, similar to LHC and RHIC, and we will examine these limiting effects later. Then at the Laslett tuneshift, the rms beam size of the protons is $58 \mu \mathrm{m}$, and for $\beta^{*}=10 \mathrm{~cm}$, the normalized rms emittance is $0.54 \mu \mathrm{m}$. Note that at $\mathrm{N}_{\mathrm{e}}=1.1 \times 10^{10}$, the beam-beam tuneshift is 0.0068 . These parameters yield luminosity equal to $5.7 \times 10^{32} \mathrm{~cm}^{-2} \mathrm{sec}^{-1}$.

\section{2. $5 \mathrm{GeV}$ electrons on $50 \mathrm{GeV}$ protons}

Following similar arguments for the case of $5 \mathrm{GeV}$ electrons on $50 \mathrm{GeV}$ protons, we arrive at the two sets of parameters outlined in Table 1. Note that luminosity at the $10^{33}$ level is attainable at these energies, for average current in the linac of $0.264 \mathrm{~A}$ and average current in the ring of $2.4 \mathrm{~A}$.

Table 1. Parameters for point designs 1 and 2.

\begin{tabular}{|c|l|l|l|l|l|}
\hline Parameter & Units & $\begin{array}{c}\text { Point } \\
\text { Design 1A }\end{array}$ & $\begin{array}{l}\text { Point } \\
\text { Design 1B }\end{array}$ & $\begin{array}{l}\text { Point } \\
\text { Design 2A }\end{array}$ & $\begin{array}{l}\text { Point } \\
\text { Design 2B }\end{array}$ \\
\hline $\mathrm{E}_{\mathrm{e}}$ & $\mathrm{GeV}$ & 3 & 3 & 5 & 5 \\
\hline $\mathrm{E}_{\mathrm{p}}$ & $\mathrm{GeV}$ & 15 & 15 & 50 & 50 \\
\hline $\mathrm{N}_{\mathrm{e}}$ & $\mathrm{ppb}$ & $1.1 \times 10^{10}$ & $1.1 \times 10^{10}$ & $1.1 \times 10^{10}$ & $1.1 \times 10^{10}$ \\
\hline $\mathrm{N}_{\mathrm{p}}$ & $\mathrm{ppb}$ & $3.0 \times 10^{10}$ & $1.0 \times 10^{11}$ & $1.0 \times 10^{11}$ & $1.0 \times 10^{11}$ \\
\hline $\mathrm{f}_{\mathrm{c}}$ & $\mathrm{MHz}$ & 150 & 150 & 150 & 150 \\
\hline$\sigma_{\mathrm{e}}^{*}$ & $\mu \mathrm{m}$ & 35 & 35 & 25 & 25 \\
\hline$\sigma_{\mathrm{p}}^{*}$ & $\mu \mathrm{m}$ & 107 & 58 & 60 & 25 \\
\hline$\varepsilon_{\mathrm{e}}^{*}$ & $\mathrm{~nm}$ & 10 & 10 & 6 & 6 \\
\hline$\varepsilon_{\mathrm{p}}^{*}$ & $\mathrm{~nm}$ & 200 & 33.6 & 36 & 6.25 \\
\hline$\beta_{\mathrm{e}}^{*}$ & $\mathrm{~cm}$ & 12 & 12 & 10 & 10 \\
\hline$\beta_{\mathrm{p}}^{*}$ & $\mathrm{~cm}$ & 6 & 10 & 10 & 10 \\
\hline$\sigma_{\mathrm{p}}^{\mathrm{p}}$ & $\mathrm{cm}$ & 6 & 10 & 10 & 10 \\
\hline$\sigma_{\mathrm{z}}^{\mathrm{e}}$ & $\mathrm{mm}$ & 1 & 1 & 1 & 1 \\
\hline$\xi_{\mathrm{p}}$ & - & .004 & .0068 & .004 & .004 \\
\hline$\Delta \mathrm{v}_{\mathrm{L}}$ & - & .004 & .05 & .004 & .024 \\
\hline $\mathrm{I}_{\mathrm{e}}$ & $\mathrm{A}$ & .264 & .264 & .264 & .264 \\
\hline $\mathrm{I}_{\mathrm{p}}$ & $\mathrm{A}$ & .720 & 2.4 & 2.4 & 2.4 \\
\hline $\mathrm{L}$ & $\mathrm{cm}{ }^{-2} \mathrm{sec}^{-1}$ & $6.2 \times 10^{31}$ & $5.7 \times 10^{32}$ & $6.2 \times 10^{32}$ & $2.1 \times 10^{33}$ \\
\hline
\end{tabular}


3. $10 \mathrm{GeV}$ electrons on $250 \mathrm{GeV}$ protons based on the RHIC storage ring [6]

The third and fourth point designs presented here are for $10 \mathrm{GeV}$ electrons colliding with $250 \mathrm{GeV}$ protons or $100 \mathrm{GeV} / \mathrm{A}$ ions using the existing RHIC storage ring. The lower energy point designs may also be implemented in RHIC, but we skip this detail.

Since the design of the Interaction Point (IP) (in particular the size of the detector) depends on the energy, it is reasonable to assume that at least two detectors will be required. The RHIC machine has two independent ion rings and thus could support one (or more) IP per ring, with collisions taking place with two different energies. The electron linac could be designed also to provide simultaneously two energies, but the detailed description of this mode will not be addressed in this report.

The presented parameters are consistent with the RHIC layout. A schematic layout of the linac-ring collider is shown in Figure 7. A schematic layout for the ring-ring scenario, also based on the RHIC accelerator, is shown in Figure 8. Table 2 summarizes the linac parameters, common to both protons and gold.

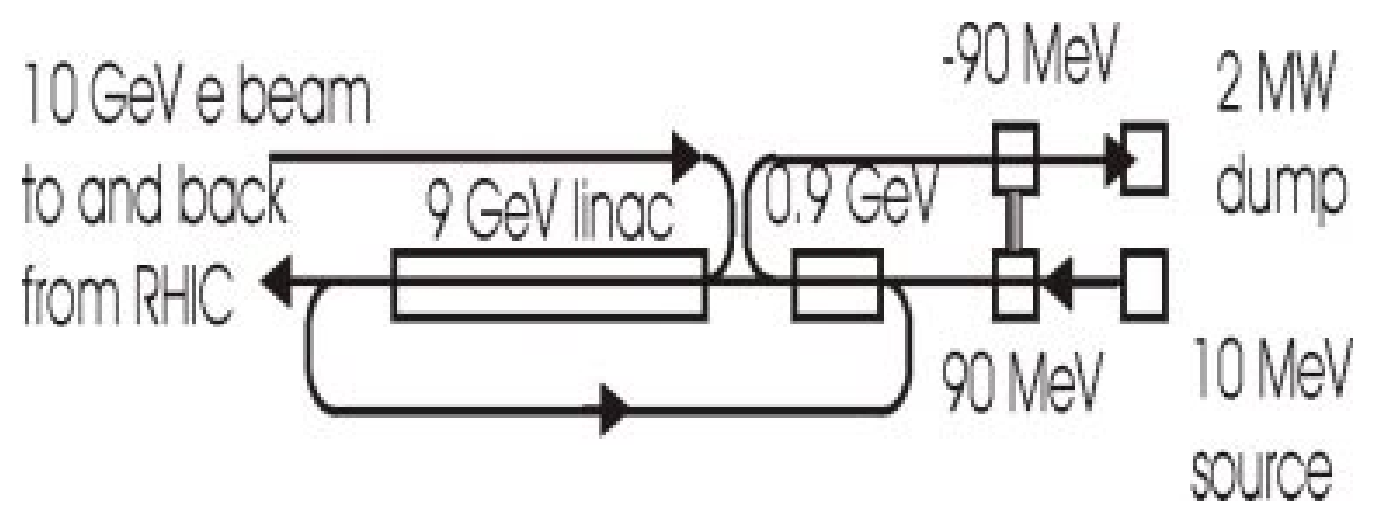

Figure 7. Schematic layout of the RHIC-based linac-ring collision scenario. (See Ref. [6]). 


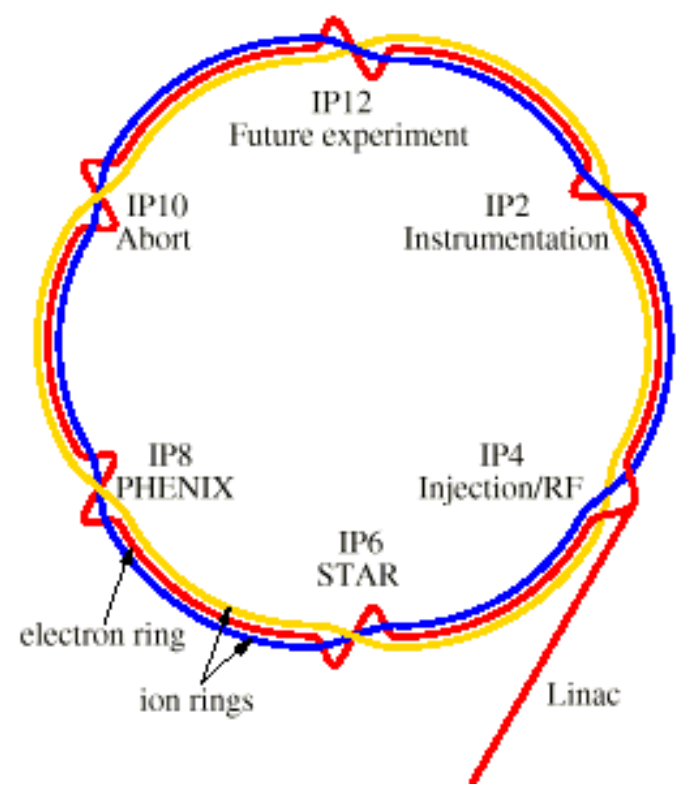

Figure 8. Schematic layout of the electron ring and the two ion rings in the RHIC-based ring-ring collision scenario (See Ref. [6]).

Table 2. Electron linac parameters, common for gold and protons.

\begin{tabular}{|l|c|c|}
\hline \multicolumn{1}{|c|}{ Parameter } & Units & Value \\
\hline Electron energy & $\mathrm{GeV}$ & 10 \\
\hline Electron average current & Amperes & 0.27 \\
\hline Collision frequency & $\mathrm{MHz}$ & 56 \\
\hline Electron bunch population $\mathrm{N}_{\mathrm{e}}$ & & $3 \times 10^{10}$ \\
\hline Electron rms emittance, $\boldsymbol{\varepsilon}_{\mathrm{e}}$ & $\mu \mathrm{m}$ & 0.003 \\
\hline
\end{tabular}

As seen in Figure 7, all the acceleration is done virtually in a straight line, to avoid emittance growth and synchrotron radiation loss in the accelerated beam. The recuperating beam is bent to return to the appropriate sections. The electron source has an injector linac that accelerates the beam to $10 \mathrm{MeV}$. The power invested (at 0.27 amperes) for this purpose is $2.7 \mathrm{MW}$. This section has no recuperation. Next is a low gradient $90 \mathrm{MeV}$ (energy gain) pre-accelerator. Here energy recuperation may be done in a dedicated linac section, and the recovered energy would then be fed through waveguides to the accelerating section, shown schematically in Fig. 7 as a connection between the accelerating and decelerating linacs. The $100 \mathrm{MeV}$ beam from the pre-accelerator is fed into a $0.9 \mathrm{GeV}$ (energy gain) intermediate linac with energy recovery done in the same section. Last is the main linac, with an energy gain of $9 \mathrm{GeV}$. The $10 \mathrm{GeV}$ beam is taken to the collision point. We do not show the details of this in Fig. 7, since the beam may be introduced into a ring-like transport 
for multiple interaction points, a single IP or anything in-between. The beam is returned at $10 \mathrm{GeV}$ to the entrance of the main linac for deceleration and energy recovery. The beam is decelerated to $1 \mathrm{GeV}$, and then sent to the intermediate energy linac for deceleration to $100 \mathrm{MeV}$. In recuperating the energy of the beam in the same linac structure we conform to the conservative limit of the Douglas principle [9] of keeping the energy ratio of the two beam under 10 . Deceleration to $10 \mathrm{MeV}$ follows in a dedicated $90 \mathrm{MeV}$ pre-dump linac.

The $10 \mathrm{MeV}$ beam is sent to the beam dump, rated at $2.0 \mathrm{MW}$ (a power level of 2 MW was demonstrated in SLAC beam dumps), assuming a synchrotron radiation loss of $0.7 \mathrm{MW}$. It is possible to decelerate the beam to a lower energy should the beam dump rating be below $2 \mathrm{MW}$, but this RF power will not be used for acceleration. Note that any synchrotron radiation power loss (anywhere in the high energy transport) will subtract from the power deposited in the beam dump. The beam dumping is done at under $10 \mathrm{MeV}$ (lower energy corresponds to synchrotron radiation losses) has very minimal activation of the beam dump. An even lower beam energy at the dump and dump power can be obtained by 'braking' the beam in a linac structure, by generating RF power that can be disposed off in dummy loads.

Tables 3 and 4 summarize the electron-gold and electron-proton collision parameters. We take the 720 bunch case, which corresponds to a bunch collision frequency of $56 \mathrm{MHz}$, and assume a minimal electron cooling of the ion beam. That results in smaller ion beam emittances and allows for larger tune shifts in the electron beam. Other than that, the RHIC parameters are mostly the same as for the ring-ring case. In the case of gold the limit is set by the beam-beam tune shift for the ions. We do not take advantage of the large possible increase in the beam-beam tune shift due to the cooling. That may account for a further increase in the luminosity.

The high-energy IP of RHIC is extremely generous in size, with free space (no accelerator component) for the detectors extending $\pm 10 \mathrm{~m}$ from the IP center. A low energy IP (using the second storage ring) can be designed with accelerator components extending much closer to the IP center, thus boosting the luminosity at low energies.

The calculation is done for an electron energy of $10 \mathrm{GeV}$, however the performance would be unaffected by a much lower (or higher) electron energy. Thermal loading in magnet chambers is not a limitation for the relatively low electron beam current. In addition, larger radii of curvature in the IP optics are possible due to the removal of spin rotation optics from that area, further reducing the thermal loads relative to a ring-ring case.

We conclude that good luminosities can be obtained using a linac-ring collider with modest electron cooling of the ions in the ring. Further increases in the luminosity are possible. In the gold case, the increase would come from pushing the Laslett tune shift to higher values, taking advantage of the cooling. In the proton case the luminosity can be improved by going to a higher current in the electron linac by pushing on the beam-beam tune shift in the ring, once again taking advantage of the cooling. 
Table 3. Electron-gold collision parameters, assuming electron cooling of RHIC

\begin{tabular}{|l|l|r|}
\hline Parameter & Units & Value \\
\hline Gold bunch population & & $1.9 \times 10^{9}$ \\
\hline Number of bunches & & 720 \\
\hline Gold 95\% normalized emittance & $\mu \mathrm{m}$ & 11 \\
\hline RMS beam size at the IP, $\sigma^{*}$ & $\mathrm{~mm}$ & 0.05 \\
\hline Electron IP beta function & $\mathrm{m}$ & 0.72 \\
\hline Electron beam-beam tune shift $\xi_{\mathrm{e}}$ & & 0.287 \\
\hline Au beam-beam tune shift $\xi_{\mathrm{i}}$ & & 0.0046 \\
\hline Ion Laslett tune shift, $\xi_{\mathrm{L}}$ & & 0.008 \\
\hline Luminosity, L & {$\left[\mathrm{cm}^{-2} \mathrm{~s}^{-1}\right]$} & $1.2 \times 10^{31}$ \\
\hline
\end{tabular}

Table 4. Electron-proton collision parameters, assuming electron cooling of RHIC

\begin{tabular}{|l|c|r|}
\hline Parameter & Units & Value \\
\hline Proton bunch population & & $0.93 \times 10^{11}$ \\
\hline Number of bunches & & 720 \\
\hline Proton 95\% normalized emittance & $\mu \mathrm{m}$ & 9.5 \\
\hline RMS beam size at the IP, $\sigma^{*}$ & $\mathrm{~mm}$ & 0.03 \\
\hline Electron IP beta function & $\mathrm{m}$ & 0.36 \\
\hline Electron beam-beam tune shift $\xi_{\mathrm{e}}$ & & 0.5 \\
\hline P beam-beam tune shift $\xi_{\mathrm{i}}$ & & 0.0046 \\
\hline P Laslett tune shift, $\xi_{\mathrm{L}}$ & {$\left[\mathrm{cm}^{-2} \mathrm{~s}^{-1}\right]$} & 0.0046 \\
\hline Luminosity, L & $2.6 \times 10^{33}$ \\
\hline
\end{tabular}

In summary, a linac has a number of distinct advantages over a ring, some of which are specific to collisions with the RHIC beam, most of which are general. Some of the advantages are:

- A linac, in principle, avoids the limitation of the electron beam-beam tune shift inherent in a ring-ring scenario. That allows one to reduce the beam size of the ion storage ring and increase its charge per bunch considerably. However, further study of the beam-beam head-tail instability is required to determine how large an advantage truly exists in the linac-ring collider scenarios.

- A linac has a very low emittance. This leads to a small collision point beam size with a relatively large beta function, increasing luminosity and simplifying the optics of the interaction point.

- The fact that the electrons are used only once means that complicated spin rotation conditions are relaxed. Thus a linac-based collider can provide a polarized electron beam at any energy, while a storage ring is limited to a very narrow energy range. 
- The interaction point optics of the linac is much simpler (since the polarization may be prepared well in advance) thus the bending radii are larger. This removes a significant synchrotron-radiation power restriction from the maximum beam current.

- A linac can operate over a wide energy range without sacrificing performance.

- The polarization of a linac is high and can be alternated rapidly at will.

- A linac produces a naturally round beam, to match well with the RHIC beam.

- A linac can be extended to higher energies with a cost that is linear in length whereas an storage ring faces an increase in RF power that goes with the fourth power of its energy.

\section{Interaction Region}

The minimum achievable beta-function at the IP is determined by the following limitations. First, making $\beta_{i}^{*}$ below rms bunch length does not increase the luminosity and we put $\beta_{i}^{*} \geq \sigma_{z}^{i}$. Second, decrease of $\beta_{i}^{*}$ causes an increase of betafunctions in the final focus quadrupole. That brings an increase of final focus chromaticity and, consequently, an increase in the sensitivity of the machine tunes to the quadrupole settings and a loss of stability for betatron motion of particles with large momentum deviations. The final focus chromaticity per interaction point can be estimated using the following formula

$$
v^{\prime} \approx \frac{1}{\pi} \frac{F}{\beta^{*}}
$$

where $F$ is the final focus focusing distance. Usually, quad currents are stabilized better than $0.01 \%$ and the increase of machine tunes sensitivity to the quadrupole settings does not cause a problem. Although the total machine chromaticity can be compensated in arcs, the chromaticity of beta-functions due to final focus normally cannot. That limits the total maximum tune shift to about 0.2 per IP. Choosing the energy spread at $5 \sigma$ to be $5 \cdot 10^{-3}$ we obtain that $v^{\prime}=\Delta v /(\Delta p / p) \approx 40$. For the focal distance of $6 \mathrm{~m}$ we obtain $\beta_{i}^{*} \approx 5 \mathrm{~cm}$. Third, the aperture is limited at the final focus quadrupoles. If superconducting quads are used at the IP, the aperture limitation is not a problem for the case with the electron cooling because of sufficiently small emittance, but it limits the beta-functions at the IP to about $10 \mathrm{~cm}$ for the case

without cooling. Figure 9 shows an example of the interaction region with $\beta_{i}^{*}=6 \mathrm{~cm}$. Only one parasitic collision occurs per bunch crossing. 


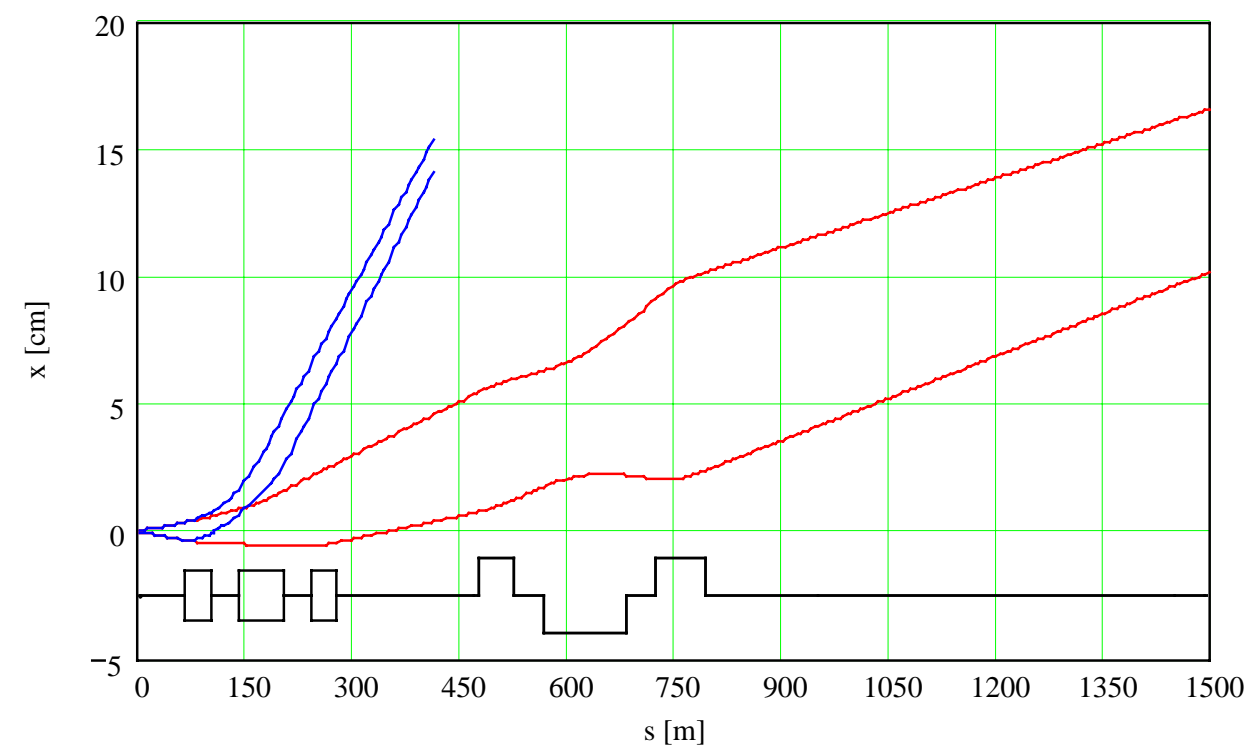

Figure 9. Example of the interaction region design for $\beta^{*}=6 \mathrm{~cm}$.

\section{Accelerator Physics Issues Of The Proton Ring}

We will examine transverse and longitudinal intrabeam scattering and collective effects. Estimates of the emittance growth of the electron beam due to collisions with the protons, although not a proton issue, will also be given here, as it may impose a limit on the proton bunch population.

a) Intrabeam Scattering (IBS)

The diffusion time for transverse IBS is given by

$$
\tau_{t r}=\frac{2 \pi}{\Lambda} \gamma_{p}^{3} \beta_{p} \frac{R^{2}}{r_{p}^{2}}\left(\frac{\varepsilon_{x}}{\beta_{a v}}\right)^{5 / 2} \frac{\sigma_{z}}{c} \frac{1}{N_{p}}
$$

where $\Lambda$ is the Coulomb logarithm defined by:

$$
\Lambda=\frac{\pi}{2} \ln \left[\gamma_{p} \sqrt{\frac{\pi}{2} \frac{\varepsilon_{x}^{3 / 2}}{r_{p} \beta_{a v}^{1 / 2}}}\right] .
$$


At $15 \mathrm{GeV}$ for the parameters of design $1 \mathrm{~A}$, the diffusion time is approximately 9 hours, which is reasonable. For design $1 \mathrm{~B}$ the rate is $79 \mathrm{sec}$, which implies that electron cooling with cooling rate of similar magnitude would be required. It is expected that cooling rates of order $1 \mathrm{~min}$ are feasible, therefore this number may be acceptable. At $50 \mathrm{GeV}$ the diffusion rate is $\sim 160$ hours for design $2 \mathrm{~A}$ and $\sim 20$ minutes for design $2 \mathrm{~B}$.

The longitudinal IBS imposes more stringent requirements, especially on the cooling rate. The expression for the longitudinal IBS diffusion rate is

$$
\tau_{\text {long }}=\frac{8 \gamma_{p}^{3} \sigma_{z} \varepsilon_{x}^{3 / 2}\left(\sigma_{E} / E\right)^{2}}{c r_{p}^{2} N_{p} \Lambda} \sqrt{\frac{C}{2 \pi v_{x}}}
$$

For $15 \mathrm{GeV}$ protons and an rms energy spread equal to $1.5 \times 10^{-3}$ the rate for design $1 \mathrm{~A}$ is $\sim 20$ minutes, which is too short for normal operating conditions. For design $1 \mathrm{~B}$, the rate is $30 \mathrm{secs}$, which again might be too fast even for cooling. However one could re-optimize for higher energy spread. At $50 \mathrm{GeV}$ for an $\mathrm{rms}$ energy spread of $3 \times 10^{-3}$, the rate for design $2 \mathrm{~A}$ is 3 hours and for design $2 \mathrm{~B}$ is 15 minutes, both reasonable.

\section{b) Collective Instabilities}

We first examine the longitudinal mode coupling or microwave instability. The threshold is given by

$$
\left|\frac{\mathrm{Z}_{\square}}{n}\right|=\sqrt{\frac{\pi}{2}} \frac{Z_{0} \alpha \gamma \sigma_{\delta}^{2} \sigma_{z}}{N_{p} r_{p}} .
$$

For $\left|\frac{Z_{\square}}{n}\right| \sim 0.25 \Omega$ (from LHC [10] and the Tevatron [11]), the number of protons per bunch is limited to $\sim 6 \times 10^{12}$. The threshold for the transverse mode coupling instability, in the regime where the bunch length is greater than the beam pipe aperture, can be expressed as [12],

$$
\left|Z_{\perp}\right|=Z_{0} \frac{\pi \gamma \omega_{s} \sigma_{z}}{3 N_{p} r_{p} \beta_{a v} \omega_{0}}
$$

For $\left|Z_{\perp}\right| \sim 5 \times 10^{4} \Omega$ (scaled from LHC), the proton bunch population is limited to $\sim 1.8 \times 10^{12}$. In conclusion, both types of instabilities occur at proton bunch populations above the design parameters we chose. 
c) Electron Beam Emittance growth due to a Single Collision (G. Krafft)

A single collision disrupts the electron beam and causes emittance growth. In an energy recovering linac, the electron beam with degraded phase space has to be recirculated for energy recovery. Deceleration in the linac cavities can result in scraping and beam loss due to adiabatic antidamping. Therefore, the amount of

tolerable beam loss at the linac exit (where the beam size is largest) imposes a limit on the tolerable emittance growth due to a single collision. This, in turn, imposes a limit on the number of protons per bunch.

Let us assume that the maximum tolerable beam loss is $4 \times 10^{-6}$ which corresponds to $1 \mu \mathrm{A}$ out of $250 \mathrm{~mA}$, based on Jefferson Lab experience. Assuming a gaussian distribution of the electrons, aperture size of $7 \mathrm{~cm}$ and an average beta function in the linac of $\sim 50 \mathrm{~m}$, then the maximum rms normalized emittance, consistent with this amount of beam loss is calculated to be $800 \mu \mathrm{m}$. In the small disruption limit, the emittance growth of the electron beam due to a single collision with the proton beam of intensity $\mathrm{N}_{\mathrm{p}}$ is given by:

$$
\varepsilon_{n}^{2}=\varepsilon_{0, n}^{2}+\left(0.194 r_{e} N_{p}\right)^{2}
$$

For $\varepsilon_{0, \mathrm{n}}=60 \mu \mathrm{m}, \varepsilon_{\mathrm{n}}=800 \mu \mathrm{m}, \mathrm{N}_{\mathrm{p}}$ has to be less than $1.5 \times 10^{12}$ particles per bunch, well below our design specification.

\section{Accelerator Physics Issues Of Energy Recovering Linacs}

The list of topics that may need to be examined in an energy recovered linac (ERL) include accelerator transport, coherent synchrotron radiation, Higher Order Mode (HOM) power dissipation and Beam Breakup phenomena. The analysis of the head-tail instability, in a linear approximation, is also outlined here. We will now examine each topic in turn.

\section{a) Accelerator Transport}

Topics of longitudinal and transverse matching and beam loss may all be relevant. Depending on the bunch length requirements at the IP, longitudinal gymnastics may be necessary and it is routinely and successfully done at JLab IRFEL [13]. Transverse phase space matching into the IP is almost invariably required for good overlap to maximize luminosity, and maybe required in order to transport and energy recover the disrupted electron beam after the collisions. Adiabatic antidamping imposes additional constraints in the linac optics. Another issue specific to ERLs has to do with the dynamic range of the linac and the ability to confine two beams of different energies in the same focusing structure and it may impose a constraint on the ratio of injected to final beam energies. 
Understanding the origin of and being able to control beam loss are crucial in an ERL with the parameters outlined above. In the JLab IRFEL several indicators place an upper limit on the amount of beam loss in the recirculator to $2 \mu \mathrm{A}$ out of $5 \mathrm{~mA}$. This amount of loss, although extremely small is unacceptable for the ERL designs discussed here, as it can potentially give rise to hundreds of $\mathrm{kW}$ of uncontrolled, lost beam power. More work is required to understand both the origin of the loss and possible cures.

\section{b) Coherent Synchrotron Radiation}

When the beam travels around a bend it radiates, and when the radiation wavelength is longer than the bunch length, it radiates coherently and interacts to deteriorate the beam quality. Both transverse and longitudinal self-forces can cause emittance growth, which is potentially serious for high brightness beam quality preservation. A self-consistent, two-dimensional code has been developed [14] and is being verified against experimental data obtained from the JLab IRFEL and the CTF II Facility at CERN.

\section{c) HOM Power Dissipation}

Power in HOMs, primarily longitudinal, depends on the product of bunch charge and average current and could present a serious enough constraint that engineering choices are imposed for improved cryogenic efficiency. The power dissipated by the beam in HOMs is given by

$$
P_{d i s s}=k_{\square} Q \bar{I}
$$

For CEBAF cavities, the calculated loss factor is equal to $5.4 \mathrm{~V} / \mathrm{pC}$ for $1 \mathrm{~mm} \mathrm{rms}$ bunch length [15], therefore the power dissipated by the beam for 0.264 A average current is approximately $5 \mathrm{~kW}$ per cavity. It is important to address the question of where these losses go. A simple model was developed [16] that shows that a) the fraction of the power dissipated on the cavity walls is a strong function of bunch length and b) most of the power is actually extracted into loads, or propagates out of the cavity through the beam pipe openings. With reasonable assumptions for the extraction efficiency of the HOM power (Q 2000), a small fraction of the power, of the order of a few Watts, is expected to be deposited on the walls, for the parameters quoted here. Engineering studies on HOM cooled absorbers between cavities or cryomodules are recommended.

\section{d) Beam Breakup}

$\mathrm{BBU}$ refers to a variety of collective phenomena that can limit the performance of srf energy recovering linacs. These coherent effects include single-bunch, singlepass phenomena which limit the charge per bunch, and multi-bunch phenomena which limit the average current. Single bunch effects include energy spread induced by variation of the longitudinal wakefield across the bunch, and emittance growth 
induced by transverse wakefields across the bunch. The induced relative energy spread at $3 \mathrm{GeV}$ and $0.264 \mathrm{~A}$ average current is $5 \times 10^{-4}$. The single bunch BBU emittance growth was discussed earlier and we concluded that it does not become an issue until the electron bunch population is increased by approximately an order of magnitude.

Multipass, multi-bunch BBU occurs when a recirculating beam through a linac cavity leads to a transverse instability. Transverse beam displacement on successive recirculations can excite HOMs that further deflect the initial beam. The recirculated beam and cavities form a feedback loop, which, for beam current greater than the threshold current of the instability, can be driven unstable. The effect is worse in srf cavities because of the higher Q's of the HOMs. The threshold current depends on various cavity and lattice parameters, including the Q's, frequencies and R/Q's of the HOMs, the beam energy, the beta functions and phase advance in both planes and the recirculation path length.

A two-dimensional simulation code, TDBBU [17] has been developed for the calculation of the threshold current in an actual machine configuration. Recent TDBBU simulations of an ERL with $10 \mathrm{MeV}$ injection energy, $5 \mathrm{GeV}$ final energy, average beta functions in the linac of approximately $15 \mathrm{~m}$ in both planes $(60 \mathrm{~m}$ maximum) and HOM data from the 9-cell TESLA cavities [18], resulted in a threshold current of about $225 \mathrm{~mA}$ [19]. Furthermore, the typical growth rate of the instability just above threshold is in the msec range, allowing for the possibility of controlling the instability with feedback.

\section{e) Beam-beam induced Head-Tail Instability (V. Lebedev)}

For the case when the electron bunches are much shorter than the proton bunches, one can make a simple estimate of the effect in the linear approximation. To find the corresponding transverse impedance one can consider that a small fraction of the proton beam of length $\Delta s$ is displaced by $x$. This will deflect the electron beam by the angle equal to

$$
\Delta \theta_{e}=\frac{Z r_{e}}{\gamma_{e} \sigma_{i}^{* 2}} \frac{d N_{i}}{d s} x \Delta s
$$

where $d N / d s$ is the charge per unit length in the proton beam. Then, the electron beam starts performing a betatron oscillation in the field of proton beam. For sufficiently small $\xi_{e}$ one can neglect this reverse attraction for the electron bunch and consider the electron bunch moving along a straight line, but this increasing distance between centers of electron and proton bunches causes a deflection of protons in the bunch tail by an angle 


$$
\Delta \theta_{i}(s)=\frac{Z r_{p} N_{e}}{\gamma_{p} \sigma_{e}^{* 2}}\left(s-s^{\prime}\right) \Delta \theta_{e}=\frac{Z^{2} r_{e} r_{p} N_{e}}{\gamma_{e} \sigma_{i}^{* 2} \gamma_{i} \sigma_{e}^{* 2}} \frac{d N_{i}}{d s}\left(s-s^{\prime}\right) x \Delta s
$$

This yields the following expressions the transverse wake function,

$$
W(s)=\frac{r_{e} N_{e}}{\gamma_{e} \sigma_{i}^{* 2} \sigma_{e}^{* 2}} s,
$$

and the transverse impedance,

$$
Z_{\perp}(s)=i \frac{Z_{0}}{4 \pi} \frac{r_{e} N_{e}}{\gamma_{e} \sigma_{i}^{* 2} \sigma_{e}^{* 2}} \frac{c^{2}}{\omega^{2}}
$$

where $Z_{0}=377 \Omega$. The threshold for the strong head-tail instability can be written as

$$
N_{t h} \approx \frac{16 \pi \gamma_{i} \sigma_{s i} \nu_{s}}{r_{p} Z^{2} \operatorname{Im}\left\langle\beta Z_{\perp}\left(c / \sigma_{s i}\right)\right\rangle} .
$$

Combining the above two formulas one finally obtains the limitation:

$$
D_{e} \xi_{i} \leq 4 v_{s}
$$

where

$$
\xi_{i}=\frac{Z r_{p} N_{e} \beta_{i}^{*}}{4 \pi \gamma_{i} \sigma_{e}^{* 2}}
$$

is the betatron tune shift of the ion beam and

$$
D_{e}=\frac{Z r_{e} N_{i} \sigma_{s i}}{\gamma_{e} \sigma_{i}^{* 2}}
$$

is the electron beam disruption parameter.

Eq. (25) can impose a severe limit on possible collider parameters and luminosity estimates in this limit were given at the beginning of this report. Nevertheless, one needs to keep in mind that the estimate was obtained in the linear approximation while the actual transverse field of the bunch is strongly non-linear across the bunch. Although it cannot eliminate the instability, it should move the threshold to higher 
beam currents. Note that the estimate itself is not good enough to claim accuracy better than factor of two. An independent study using a two-particle model arrived at the same eq. (25) within a factor of two [20]. Performing more detailed study and computer simulations of the instability is going to be one of the high priority tasks at Jefferson Lab in the near future. We also note that the proton bunch is quite long and a transverse feedback system for suppression of the instability's lowest modes may be feasible, which can increase the instability threshold by a factor of 2 or 3 . Thus, we currently believe that additional increase in the instability threshold by a factor of 3-10 is possible.

\section{Technological Issues}

\section{a) High current source of polarized electron}

The generation of high average current, high polarization electron beams is a significant technological issue. The state-of-the art in polarized electron sources was reviewed in the PAC99 article by Sinclair [21]. The prospects for sources of high average current polarized electrons was presented in the Proceedings of the $2^{\text {nd }}$ eRHIC Workshop [22]. Presently, polarized sources at JLab have cathode operational lifetimes one order of magnitude greater than those reported by Sinclair at PAC99. Cathode operational lifetime in these sources is limited only by ion backbombardment, and now exceeds 100,000 Coulombs $/ \mathrm{cm}^{2}$. While construction of a high average current polarized source with modest polarization $(\sim 37 \%)$ is probably within reach, a source with a high polarization $(\sim 75 \%)$ faces a number of serious technological challenges. Significant R\&D would be required before one could plan on a source delivering a high average current at high polarization.

\section{b) Electron Cooling}

This is an important topic, which is mentioned here only for completeness. It is thoroughly addressed in a separate section of this report.

\section{Concluding Remarks And Outlook}

Preliminary results of a feasibility study of an energy recovering electron linac on a proton ring collider are presented. Luminosities at the $10^{33}$ level appear attainable and the linac-on-ring scenario presents a significant advantage with respect to spin manipulations, energy variability and synchrotron radiation power loading of the detectors.

R\&D topics that would be required before such a facility is designed and built have been identified and include:

1. High current polarized electron source

2. High current $(\sim 100 \mathrm{~mA})$ demonstration of energy recovery

3. Theoretical and, if possible, experimental investigation of the beam-beam induced head-tail instability and feedback 
4. Electron cooling and its ramifications on Laslett and beam-beam tuneshifts

5. Development of multipass BBU feedback

Recently, recirculating, energy-recovering linacs have attracted much attention and are being considered for a number of applications, such as drivers for synchrotron radiation sources, and high average power FELs. A number of the listed R\&D topics, especially those related to the energy recovery of high average currents, are being pursued by these communities, so it is safe to assume that progress will be rapid in these directions.

\section{REFERENCES}

1. T. I. Smith, et al., "Development of the SCA/FEL for use in Biomedical and Materials Science Experiments," NIM A259 (1987) 1-7

2. D. W. Feldman et al., "Energy Recovery in the Los Alamos Free Electron Laser," NIM A259 (1987) 26-30

3. N. R. Sereno, University of Illinois, Ph.D. Thesis (1994)

4. G. R. Neil, et al., "Sustained Kilowatt Lasing in a Free Electron Laser with Same-Cell Energy Recovery," Physical Review Letters, Volume 84, Number 4 (2000)

5. I. A. Koop, et al., "Conceptual Design Study of the Electron-Proton Storage Ring Collider with Polarized Beams," this document and Proc. of the $2^{\text {nd }}$ EPIC Workshop, MIT, September 2000

6. I. Ben-Zvi, J. Kewisch, J. Murphy and S. Peggs, "Accelerator Physics Issues in eRHIC," Proc. of the $2^{\text {nd }}$ eRHIC Workshop, Yale University, April 2000

7. P. Kneisel, "Preliminary Experience with "In-Situ" Baking of Niobium Cavities," Proc. of RF Superconductivity Workshop, Santa Fe, November 1999.

8. A. W. Chao, B. Richter and C.-Y. Yao, "Beam Emittance Growth Caused by Transverse Deflecting Fields in a Linear Accelerator," NIM 178 (1980) 1-8

9. D. Douglas, Jefferson Lab Technical Note, TN-98-040, 1998

10. L. Vos, Private communication

11. K.-Y. Ng, Private communication

12. A. W. Chao, "Physics of Collective Beam Instabilities in High Energy Accelerators," Wiley Series in Beam Physics and Accelerator Technology, 1993

13. G. A. Krafft and D. Douglas, Private communication

14. R. Li, "The Impact of Coherent Synchrotron Radiation on the Beam Transport of Short Bunches," Proc. of the 1999 Particle Accelerator Conference, New York, 1999

15. B. Yunn, Private communication

16. L. Merminga, et al., "Specifying HOM-power Extraction Efficiency in a High Average Current, Short Bunch Length SRF Environment," Proc. of Linac 2000 Conference, Monterey, August 2000

17. G. A. Krafft and J. J. Bisognano, "Two dimensional simulations of multipass beam breakup," Proc. of 1987 Particle Accelerator Conference, pg. 1356

18. J. Sekutowicz, "Higher Order Mode Coupler for TESLA," DESY Report, TESLA 94-07, February 1994

19. I. Bazarov, Private communication

20. R. Li and J. Bisognano, Private communication

21. C. K. Sinclair, "Recent Advances in Polarized Electron Sources," Proc. of the 1999 Particle Accelerator Conference, New York, 1999

22. P. Hartmann et al., "Polarized Electron Linac Sources," Proc. of the $2^{\text {nd }} e$ RHIC

Workshop, Yale University, April 2000 